\title{
BMJ Open Interaction of diabetes and smoking on stroke: a population-based cross- sectional survey in China
}

\author{
Heqing Lou, ${ }^{1}$ Zongmei Dong, ${ }^{1,2}$ Pan Zhang, ${ }^{2}$ Xiaoping Shao, ${ }^{1}$ Ting Li, ${ }^{2}$ \\ Chunyan Zhao, ${ }^{1}$ Xunbao Zhang, ${ }^{1}$ Peian Lou ${ }^{1,2}$
}

To cite: Lou H, Dong Z, Zhang $\mathrm{P}$, et al. Interaction of diabetes and smoking on stroke: a populationbased cross-sectional survey in China. BMJ Open 2018;8:e017706. doi:10.1136/ bmjopen-2017-017706

- Prepublication history for this paper is available online. To view these files, please visit the journal online (http://dx.doi. org/10.1136/bmjopen-2017017706).

Received 12 May 2017 Revised 23 January 2018 Accepted 1 March 2018
Check for updates

${ }^{1}$ The School of Public Health, Xuzhou Medical University, Xuzhou, China

${ }^{2}$ Department of Noncommunicable Disease Control, Xuzhou Center for Disease Control and Prevention, Xuzhou, China

Correspondence to Professor Xunbao Zhang; xunbz@163.com

\section{ABSTRACT}

Objectives Diabetes and smoking are known independent risk factors for stroke; however, their interaction concerning stroke is less clear. We aimed to explore such interaction and its influence on stroke in Chinese adults. Design Cross-sectional study.

Setting Community-based investigation in Xuzhou, China. Participants A total of 39887 Chinese adults who fulfilled the inclusion criteria were included.

Methods Participants were selected using a multistage stratified cluster method, and completed self-reported questionnaires on stroke and smoking. Type 2 diabetes mellitus (DM2) was assessed by fasting blood glucose or use of antidiabetic medication. Interaction, relative excess risk owing to interaction (RERI), attributable proportion (AP) and synergy index (S) were evaluated using a logistic regression model.

Results After adjustment for age, sex, marital status, educational level, occupation, physical activity, body mass index, hypertension, family history of stroke, alcohol use and blood lipids, the relationships between DM2 and stroke, and between smoking and stroke, were still significant: ORs were 2.75 (95\% Cl 2.03 to 3.73$)$ and 1.70 (95\% Cl 1.38 to 2.10), respectively. In subjects with DM2 who smoked, the RERI, AP and S values (and 95\% Cls) were 1.80 (1.24 to 3.83), 0.52 (0.37 to 0.73 ) and 1.50 (1.18 to 1.84), respectively.

Conclusions The results suggest there are additive interactions between DM2 and smoking and that these affect stroke in Chinese adults.

\section{INTRODUCTION}

Stroke is an ongoing global health problem. In 2016, there were 5.53 million people who suffered from stroke worldwide. Stroke was also the second most common cause of premature mortality and secondary disability. ${ }^{1}$ The 2015 Report on Chinese Stroke Prevention indicates stroke as the leading cause of death in China, with incidence increasing by $8.7 \%$ per annum. ${ }^{2}$ Stroke rates in China are higher than those in Western and other Asian countries. ${ }^{34}$ Moreover, the number of patients with stroke in China is likely to increase because of lifestyle and demographic changes, and inadequate control of major risk factors for

\section{Strengths and limitations of this study}

The strengths of this study were that a large sample population was randomly selected from the general population of Xuzhou and many confounding risk factors were adjusted for.

- Owing to the cross-sectional design, we could not determine a causal combined relationship among diabetes, smoking and stroke.

- We were not able to control for some important and well-known risk factors of diabetes, such as heart rate and cardiovascular causes.

- We did not measure fresh fruit consumption, which is causally related to stroke.

stroke. ${ }^{2}$ It is therefore important to identify and prevent these risk factors.

The major risk factors for stroke are hypertension, diabetes mellitus, hypercholesterolaemia, smoking, physical inactivity, obesity and a family history of stroke. ${ }^{5-8}$ Although these play a role in the development of stroke, its formation is not entirely caused by a single risk factor. The more risk factors a person has, the greater the likelihood of incurring a stroke. ${ }^{7}$ In fact, $>90 \%$ of the global burden of stroke in 2013 was attributable to the combined effect of all modifiable risk factors. ${ }^{8}$

People with comorbid diabetes and smoking may represent a subgroup with high risk of developing stroke; however, few studies have examined the interaction of diabetes and smoking with regard to stroke. The primary aim of this cross-sectional study was to examine the interaction of type 2 diabetes mellitus (DM2) and smoking on stroke in Chinese adults. A secondary aim was to evaluate the associations between DM2 and stroke, and between smoking and stroke.

\section{MATERIALS AND METHODS}

Study design and recruitment criteria

This population-based, cross-sectional survey was conducted in Xuzhou City, Jiangsu 
Province, China, from February to June 2013. The sample was selected with two-stage probability proportional to size, from all 11 regions in Xuzhou. In the first stage, five subdistricts/townships in urban/rural areas were selected in accordance with the population of each subdistrict/ township from each region, with probability proportional to size sampling. In the second stage, five communities/ villages were selected in accordance with the population of each community/village from each subdistrict/township with probability proportional to size sampling. In the final stage, one person aged $\geq 18$ years and who had lived in his or her current residence for $\geq 5$ years was selected from each household through use of a Kish selection table. Those who met either or both of the following criteria were excluded: (1) previous diagnosis of neuropathy, psychosis or unclear speech or (2) member of the floating population or temporary residents. A total of $\geq 13500$ people were selected, assuming an estimation incidence of stroke of $2.0 \%,{ }^{9}$ with $90 \%$ power, $\alpha=0.05$ and allowing for a dropout rate of $15 \%$. Trained interviewers interviewed participants face-to-face on the day of the participants' regular medical appointments. All participants underwent 12 hours overnight fasting and blood sampling to test basic fasting plasma glucose. After blood sampling, each received a health examination and completed a structured questionnaire inquiring on demographic information, medical history, medication history and smoking, alcohol consumption and exercise habits.

\section{Key measurements}

Stroke was assessed using subjects' self-reported responses, and defined as an acute disturbance of focal areas in the brain lasting for $\geq 24$ hours and thought to be caused by intracranial haemorrhage or ischaemia. ${ }^{10}$ We examined the medical records of participants reporting a diagnosis of stroke to check that participants satisfied this definition. The diagnosis was also confirmed through CT and MRI scans. Detailed clinical information about stroke was based on the International Classification of Disease, 10th Revision, codes I60-I64.

DM2 was defined as fasting blood glucose $\geq 7.0 \mathrm{mmol} / \mathrm{L}$, any use of antidiabetic medication or self-reported history of DM2. ${ }^{11}$ Hypertension was defined as systolic blood pressure $\geq 140 \mathrm{~mm} \mathrm{Hg}$ or diastolic blood pressure $\geq 90 \mathrm{~mm} \mathrm{Hg}$, any use of antihypertensive medication or self-reported history of hypertension. ${ }^{12}$

\section{Covariates}

Age, sex, current employment status, marital status, level of education, cigarette smoking, alcohol consumption, physical activity and family history of diseases, including DM2, hypertension and stroke, were assessed using a standardised questionnaire. Employment status was categorised as manual, non-manual, unemployed or retired. Education was categorised as below high school, high school or above high school. Lifestyle variables included cigarette smoking, alcohol consumption and physical activity level. Cigarette smoking was defined as having smoked at least 100 cigarettes in one's lifetime. Information was obtained on the amount and type of alcohol consumed during the previous year, and alcohol drinking was defined as consumption of $\geq 30 \mathrm{~g}$ / week for $\geq 1$ year. Regular leisure-time physical activity was defined as participation in moderate or vigorous activity for $\leq 30 \mathrm{~min} /$ day, $\geq 3$ days a week. Each participant's height (to the nearest $0.1 \mathrm{~cm}$ ) and weight (to the nearest $0.1 \mathrm{~kg}$ ) in light indoor clothing were measured. Body mass index (BMI in $\mathrm{kg} / \mathrm{m}^{2}$ ) was calculated; categorised as underweight $\left(<18.5 \mathrm{~kg} / \mathrm{m}^{2}\right)$, normal weight $\left(18.5-24.0 \mathrm{~kg} / \mathrm{m}^{2}\right)$ or overweight/obese $\left(>24.0 \mathrm{~kg} / \mathrm{m}^{2}\right) .{ }^{13}$ Dyslipidemia was defined as use of any lipid-lowering medication or self-reported history of the condition.

\section{Statistical analysis}

Participants were divided into four groups in accordance with their smoking status and DM2: non-smokers with no DM2, smokers with no DM2, non-smokers with DM2 and smokers with DM2. Statistical analysis was performed using IBM SPSS for Windows, V.16.0 (SPSS Chicago, Illinois, USA). The general characteristics of continuous variables were compared across the four subgroups using analysis of variance. The categorical variables were expressed as a percentage and the groups were compared using a $\mathrm{X}^{2}$ test. Logistic regression analysis was performed to estimate the probability of having a stroke and $95 \%$ CI for each risk factor category stratified by DM2 and smoking, adjusting for age, sex, occupation, education, marital status, BMI, physical activity, drinking status, hypertension status and family history of disease, including DM2, hypertension and stroke.

Biological interactions should be based on an additive scale rather than a multiplication scale. ${ }^{145}$ We therefore used three measures to estimate biological interactions between DM2 and smoking: relative excess risk owing to interaction (RERI), attributable proportion (AP) owing to interaction and synergy index (S). RERI is the excess risk attributed to interaction relative to the risk without exposure to diabetes and smoking. AP refers to the attributable proportion of disease caused by interaction in subjects with exposure to both variables. $\mathrm{S}$ is the excess risk from exposure to both variables when there is a biological interaction relative to the risk from exposure to both variables without interaction. In the absence of additive interactions, RERI and AP equal $0 .{ }^{1416}$ In the current study, RERI $>0, A P>0$ and $S>0$ indicated statistical significance, set at $\mathrm{P}<0.05$ (two-tailed).

\section{Ethics approval and consent to participate}

The procedures followed were in accordance with the standards of the ethics committee of the Xuzhou Center for Disease Control and Prevention and with the Declaration of Helsinki (1975, revised 2000). Written informed consent was obtained from all participants.

\section{RESULTS}

\section{General characteristics of participants}

Of the 41658 individuals initially sampled, 1253 did not respond to the smoking question or complete the blood 
Table 1 General characteristics of diabetes and smoking in the study population $(n=39887)$

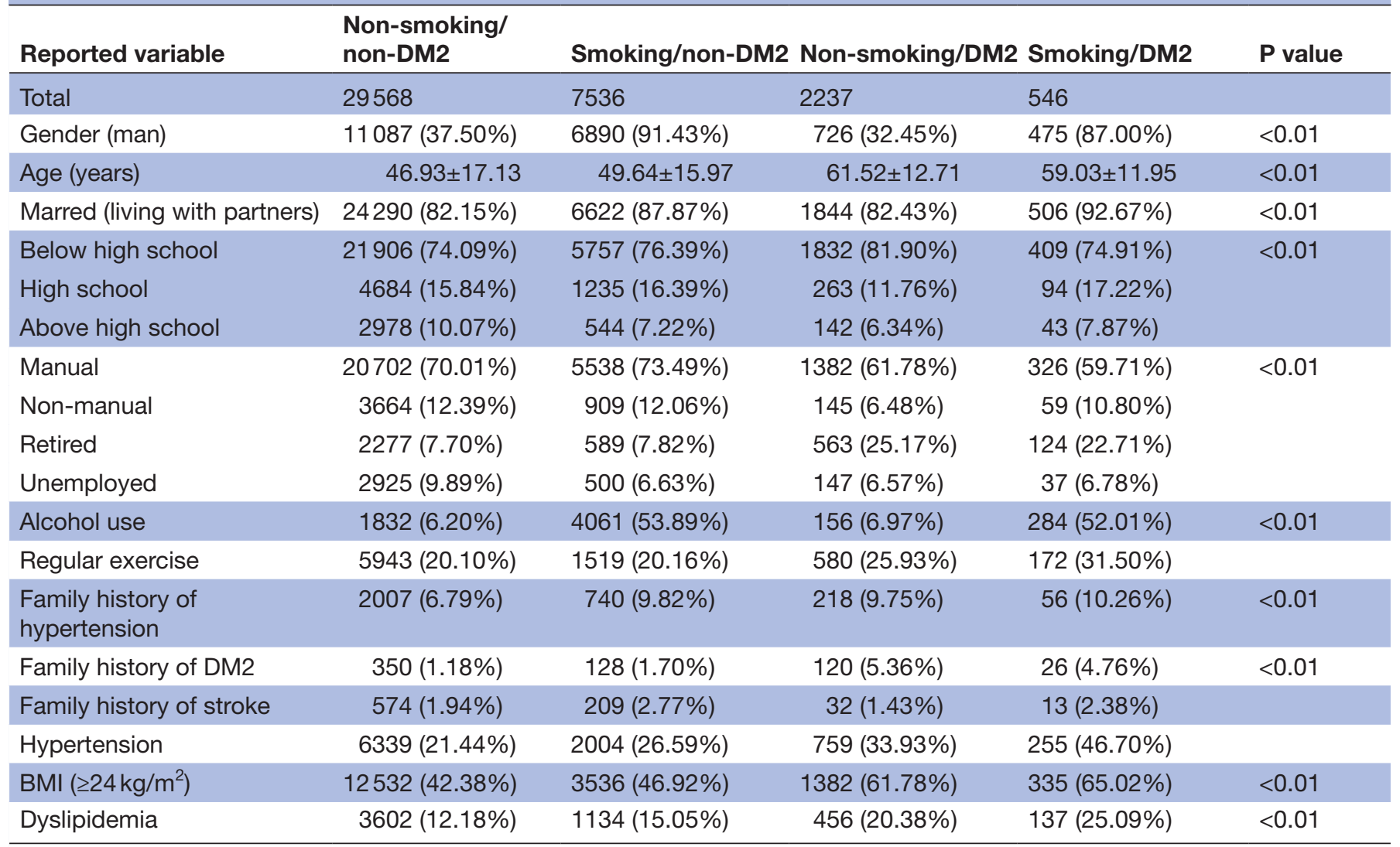

BMI, body mass index; DM2, type 2 diabetes mellitus.

glucose tests, and 518 did not meet our study criteria. A final total of 39887 adults (19178 men and 20709 women) with complete data were included in the analysis (response rate: $95.7 \%$ ). Table 1 shows the characteristics of the study population. The proportion of participants with DM2 was $7.00 \%$ (2783/39 887), of subjects with stroke with DM2 was $16.67 \%$ and of subjects without stroke with DM2 was only $6.75 \%$; there was a statistically significant difference between the two groups $\left(\chi^{2}=135.92\right.$, $\mathrm{P}<0.001)$. The proportion of smokers was $20.26 \%$ (8082/39 887), participants with stroke who smoked was $32.24 \%$ and participants without stroke who smoked was $19.98 \%$; there was a statistically significant difference in smoking between patients with stroke and without stroke $\left(\chi^{2}=83.49, \mathrm{P}<0.001\right)$.

Association of diabetes and smoking with stroke

The $5.50 \%$ incidence of stroke in subjects with DM2 exceeded the $2.06 \%$ incidence in those with no DM2 $\left(\chi^{2}\right.$ $=139.11, \mathrm{P}<0.001)$. Individuals who smoked had a higher stroke incidence compared with non-smokers $(3.36 \%$ vs $1.96 \% ; \chi^{2}=83.49, \mathrm{P}<0.001$; see table 2 ). Subjects with DM2 had a significantly increased risk of stroke compared with those with no DM2 (OR 2.65, 95\% CI 1.70 to 4.41, $\mathrm{P}<0.001$ ), after adjusting for confounders (see table 3 ). The risks of ischaemic and haemorrhagic stroke were

Table 2 Associations between smoking, diabetes and stroke

\begin{tabular}{|c|c|c|c|c|c|}
\hline Variables & Stroke & Non-stroke & $\begin{array}{l}\text { Unadjusted } \\
\text { OR }(95 \% \mathrm{CI})\end{array}$ & $\begin{array}{l}\text { Adjusted OR } \\
(95 \% \mathrm{Cl})\end{array}$ & $P$ value \\
\hline \multicolumn{6}{|l|}{ Smoking } \\
\hline No & 296 (32.24\%) & 7786 (19.98\%) & & & \\
\hline \multicolumn{6}{|c|}{ Type 2 diabetes mellitus } \\
\hline
\end{tabular}

Adjusted for age, sex, education, marriage status, employment, family history of diabetes, family history of hypertension, family history of stroke, alcohol consumption, physical activity, hypertension, diabetes or smoking, body mass index and lipids. 
Table 3 ORs for the association between smoking and stroke by diabetes among participants

\begin{tabular}{|c|c|c|c|c|c|c|}
\hline Smoking & Diabetes & No stroke & Stroke & $\begin{array}{l}\text { Unadjusted } \\
\text { OR }(95 \% \mathrm{Cl})\end{array}$ & Adjusted OR (95\% CI) & $P$ value \\
\hline No & No & 29056 & 512 & 1 & 1 & \\
\hline \multirow[t]{2}{*}{ Yes } & No & 7283 & 253 & 1.97 (1.54 to 2.37$)$ & 1.65 (1.36 to 2.00$)$ & $<0.01$ \\
\hline & Yes & 503 & 43 & 4.85 (2.65 to 6.21$)$ & 3.45 (2.30 to 5.16$)$ & $<0.01$ \\
\hline
\end{tabular}

Adjusted for age, sex, education, marriage status, employment, family history of diabetes, family history of hypertension, family history of stroke, alcohol consumption, physical activity, hypertension, body mass index and lipids.

increased by DM2, after adjusting for confounders; the ORs were 2.71 (95\% CI 1.72 to 4.49$)$ and $1.82(95 \%$ CI 1.34 to 3.35 ), respectively. Smokers had a significantly increased risk of stroke compared with non-smokers (OR $1.83,95 \%$ CI 1.59 to $2.14, \mathrm{P}<0.001$ ), after adjusting for confounders (see table 3). The risks of ischaemic and haemorrhagic stroke were increased by smoking, after adjusting for confounders; the ORs were 1.32 (95\% CI 1.12 to 2.53 ) and 1.95 (95\% CI 1.40 to 3.41 ), respectively.

\section{Interaction between diabetes and smoking in relation to stroke}

Individuals who only had DM2 or only smoked had a significantly increased risk of stroke compared with those who did not have DM2 and did not smoke (OR 2.00, 95\% CI 1.56 to 2.56; OR 1.65, 95\% CI 1.36 to 2.00 ; respectively; all $\mathrm{P}<0.001$ ), after adjusting for confounders. Table 3 shows the results from the multiple logistic regression models. The incidence of stroke was greatest in those who had DM2 and smoked (OR 3.45, 95\% CI 2.30 to 5.16, $\mathrm{P}<0.001$ ), after adjusting for confounders.

\section{Sensitivity analysis}

There was a strong additive interaction between DM2 and smoking (RERI 1.80, 95\% CI 1.24 to 2.14; AP 0.52, 95\% CI 0.37 to 0.73 and S $1.50,95 \%$ CI 1.18 to 1.84 , respectively); $52 \%$ of stroke occurrence was attributed to the interaction between DM2 and smoking (table 4).

\begin{tabular}{l}
$\begin{array}{l}\text { Table } 4 \text { Measures for estimating biological interaction } \\
\text { between smoking and diabetes for prevalence of stroke in } \\
\text { participants }\end{array}$ \\
$\begin{array}{l}\text { Measures of biological } \\
\text { interaction }\end{array}$ \\
\hline $\begin{array}{l}\text { Relative excess risk owing to } \\
\text { interaction }\end{array}$ \\
$\begin{array}{l}\text { Attributable proportion } \\
\text { Synergy index }\end{array}$ \\
\hline
\end{tabular}

Reference group is no smoking with non-diabetes.

Adjusted for age, sex, education, marriage status, employment, family history of diabetes, family history of hypertension, family history of stroke, alcohol consumption, physical activity, hypertension, body mass index and lipids.

\section{DISCUSSION}

There were two main findings in this study. First, there was an interaction of DM2 and smoking with relation to the incidence of stroke. Second, DM2 and smoking were found to be significantly associated with increased risk of stroke in Chinese adults, independent of potential confounders such as age, sex, occupation, education, marital status, BMI, physical activity, drinking status, hypertension status and family history of diseases including diabetes, hypertension or stroke.

The proportion of participants with DM2 in this study was $19.67 \%$, which is lower than that reported in the China National Stroke Registry Study (27.0\%), ${ }^{17}$ lower than that reported in a comparison of risk factors for ischaemic stroke in Chinese versus Caucasian individuals $(25.0 \%),{ }^{18}$ and higher than that reported in a study of the Chinese National Health Insurance programme $(3.8 \%)^{19}$ and a review of stroke in China $(4 \%-15 \%) .{ }^{20}$ The present results are also inconsistent with figures for diabetes in patients with stroke reported for other countries. ${ }^{21}{ }^{22}$ This discrepancy may be due to differences in study design, sampling size, region, diabetes diagnosis and the clinical features of stroke, but could also be a result of ethnic group differences, as many studies have shown that stroke incidence rates differ among ethnic groups. ${ }^{23}$

Numerous epidemiological studies, including cross-sectional studies and prospective cohort studies, have demonstrated associations between diabetes and stroke. ${ }^{224-27} \mathrm{~A}$ population-based study of 173979 discharged patients admitted with haemorrhagic stroke was conducted in Spain from 2003 to 2012. The authors of that study reported a positive association between diabetes and stroke (incidence rate ratio $1.38,95 \%$ CI 1.35 to 1.40 for men; incidence rate ratio $1.31,95 \%$ CI 1.29 to 1.34 for women).$^{22}$ Folsom et al reported that the relative risk (RR) of ischaemic stroke was 2.22 (95\% CI 1.5 to 3.2) for individuals with diabetes after adjustment for other stroke risk factors in a follow-up period of 6-8 years. ${ }^{24}$ Iso $e t a l^{25}$ reported that the association between non-embolic ischaemic stroke and diabetes was particularly strong among non-hypertensive subjects with higher subscapular skinfold thickness values; the multivariate RR was 4.9 (95\% CI 2.5 to 9.5 ) in a 17-year prospective cohort study of 10582 Japanese individuals (4287 men and 6295 women) aged 40-69 years. A systematic review and meta-analysis 
of 64 cohort studies with 775385 individuals showed that diabetes is consistently associated with increased risk of stroke; the pooled maximum-adjusted RR of stroke associated with diabetes was 2.28 (95\% CI 1.93 to 2.69) for women and 1.83 (95\% CI 1.60 to 2.08) for men. ${ }^{26}$ Liao et al also reported that patients with diabetes have an increased risk of stroke (adjusted HR 1.75, 95\% CI 1.64 to 1.86) compared with those without diabetes; associations between diabetes and stroke risk were significant for both sexes and all age groups. ${ }^{27}$ The present findings also demonstrated an association between diabetes and stroke.

Robson $e t a t^{28}$ confirmed that poor blood sugar control increases the risk of stroke. One prospective cohort study of 467508 men and women aged 30-79 years with no history of diabetes or stroke showed that each $1 \mathrm{mmol} / \mathrm{L}$ $(18 \mathrm{mg} / \mathrm{dL})$ higher than usual increase of random plasma glucose level above $5.9 \mathrm{mmol} / \mathrm{L}(106 \mathrm{mg} / \mathrm{dL})$ was associated with ischaemic stroke (adjusted HR 1.08, 95\% CI 1.07 to 1.09$).{ }^{29}$ Moreover, a study of 4669 patients who had incurred a minor stroke revealed during a 3-month follow-up that patients with stroke and diabetes experienced stroke recurrence and disability. ${ }^{30}$ Therefore, effective glycaemic control reduces the incidence of stroke and can reduce stroke recurrence and associated disability.

A higher proportion of patients with stroke than without stroke tend to be smokers. Indeed, Wang et al reported that $48 \%$ of patients with stroke smoked. ${ }^{31}$ Tsai et $a l^{18}$ reported a figure of $38 \%$ and our results showed $32.24 \%$. Although the proportions differ, they are all quite high. The discrepancy may reflect a bias in the reporting of smoking status among study participants.

Many studies have shown that smoking is a strong risk factor for development of stroke. ${ }^{32-34}$ The British Regional Heart Study, which included 7735 men aged 40-59 years, showed that after full adjustment for other risk factors, current smokers had a nearly fourfold RR of stroke compared with never-smokers (RR 3.7, 95\% CI 2.0 to 6.9 ). Ex-cigarette smokers showed lower risk than current smokers, but showed excess risk compared with never-smokers (RR $1.7,95 \% \mathrm{CI} 0.9$ to $3.3, \mathrm{P}=0.11$ ). ${ }^{33}$ During a mean follow-up of 8.5 years, the risk for all forms of stroke significantly increased (RR $0.9-3.3,95 \%$ CI 1.4 to 24) and increased for ischaemic strokes (RR 4.8, 95\% CI 1.2 to 20) among cigarette-smoking women with a cigarette-smoking spouse compared with those with a non-smoking spouse and after adjusting for other cardiovascular risk factors. ${ }^{34}$ However, a systematic review and meta-analysis of 81 cohorts in Asia, including 3980359 individuals and 42401 strokes, showed smoking did not contribute to the risk of stroke. ${ }^{35}$ The proportion of Chinese patients with stroke who smoke is higher than that of Caucasians. ${ }^{18}$ One systematic review and meta-analysis of 15 cohort studies and 178 case-control studies found smoking was an independent risk factor for stroke (pooled RR 1.27, 95\% CI 1.21 to 1.35) in a Chinese population. ${ }^{7}$ More frequent smokers are more likely to incur a stroke. ${ }^{36}$ A meta-analysis that included 16886 men and
18539 women without known diabetes revealed haemoglobin A1c was $0.10 \%$ (95\% CI 0.08 to $0.12,1.1 \mathrm{mmol} /$ mol (0.9-1.3) ) higher in current smokers and $0.03 \%$ (95\% CI 0.01 to $0.05,0.3 \mathrm{mmol} / \mathrm{mol}(0.1-0.5)$ ) higher in ex-smokers than in never-smokers. ${ }^{38}$ Therefore, our findings support previous evidence smoking is associated with stroke in Chinese populations. ${ }^{4}$ However, the present study only wanted to observe the interaction of smoking and diabetes on stroke, the cigarette smokers were not categorised as current, former and never-smokers. Therefore, the association between smoking and stroke in our study and in other studies should be compared carefully.

One study reported that in comparison with non-smoking patients with no diabetes mellitus or hypertension, patients with those conditions and who smoked had a 3-fold increase in prevalence of peripheral vascular disease and a 3.5-fold increase in cerebrovascular disease. ${ }^{39}$ Our results reinforce such findings.

The pathophysiological mechanisms of hyperglycaemia induce oxidative stress; promote formation of advanced glycosylation end products ${ }^{40}{ }^{41}$; increase blood-brain barrier permeability and inflammatory responses ${ }^{42}$; lead to accumulation of reactive oxygen species/reactive nitrogen, inflammation and mitochondrial dysfunction $^{43}$; lead to cellular dysfunction; damage vascular tissue; inhibit endogenous vascular protective factors; alter vascular homeostasis ${ }^{44}$; raise levels of reactive oxygen species and advanced glycation end products; decrease levels of mitochondrial superoxide dismutase ${ }^{41}$ and correlate with endothelial cell dysfunction and nitric oxide production. ${ }^{45}$ All these actions contribute to accelerating the atherosclerotic process. Therefore, patients with diabetes are more prone to incurring stroke.

Cigarette smoking is associated with increased reactive oxygen species, oxidative stress, blood-brain barrier permeability, sympathetic activation and nitric oxide production, reduced cerebral blood flow and serum superoxide dismutase levels, attenuation of the vasodilation of cerebral arterioles and induction of atherosclerosis and thrombosis. ${ }^{46-49}$ Moreover, cigarette smoke elevates serum levels of advanced glycation end products and reduces soluble receptors for those end products, resulting in development of atherosclerosis and related stroke. ${ }^{50-52}$ Smoking is therefore correlated with increased risk of stroke.

Collectively, diabetes and smoking induce oxidative stress and nitric oxide production; increase reactive oxygen species, blood-brain barrier permeability and the level of advanced glycation end products and reduce cerebral blood flow and serum superoxide dismutase. Therefore, patients with diabetes who smoke also have a greater risk of stroke.

The strengths of the current study are that we used a community-based multistage sampling design, large sample size and randomly selected participants. However, the study has several limitations. First, because of the cross-sectional design, we could not determine a causal relationship between DM2, smoking and stroke. Second, 
we were unable to control for some important and well-known risk factors of stroke, such as heart rate ${ }^{53}$ and cardiac causes. ${ }^{6}$ Third, we did not measure fresh fruit consumption, ${ }^{54}$ which is causally negative related to stroke. Finally, patients self-reported their cigarette smoking status; therefore, the risk of misclassification and recall bias with the definition of smoking could not be avoided.

\section{CONCLUSION}

The results of this cross-sectional study indicate patients with diabetes who smoke are 3.5 times more likely to develop stroke than patients without diabetes who do not smoke. Diabetes and smoking had a combined positive correlation with stroke. These results have important public health implications. Among Chinese adults, the current rate of smoking is as high as $28.3 \%^{55}$ and DM2 prevalence is $11.6 \% .^{56}$ Therefore, it is important to implement stroke-prevention measures aimed at reducing smoking and improving glycaemic control in patients with diabetes in China.

Acknowledgements The authors would like to thank all the participants involved in the survey. The authors would also like to thank the Regional Centers for Disease Control and Prevention as well as clinics in Xuzhou for their collaboration.

Contributors $\mathrm{HL}$ wrote/edited the manuscript and created tables. ZD, PZ, XS, TL, $\mathrm{CZ}, \mathrm{XZ}$ and $\mathrm{PL}$ contributed to the discussion and reviewed/edited the manuscript. $\mathrm{XZ}$ conceptualised the study. PL is the guarantor of this work and, as such, had full access to all data in the study and takes responsibility for the integrity of the data and accuracy of the data analysis. All authors read and approved the final manuscript.

Funding This research was funded by the Preventive Medicine research projects of Jiangsu Province Health Department in 2015 (Y2015010) and the Science and Technology projects of Xuzhou City in 2015 (KC15SM046).

Disclaimer The researchers were independent from the funders. The study funders had no influence on the study design, data collection, analysis, interpretation of data, writing of the report or decision to submit the article for publication.

Competing interests None declared.

Patient consent Obtained.

Ethics approval The study protocol was approved by the Xuzhou Center for Disease Control and Prevention.

Provenance and peer review Not commissioned; externally peer reviewed.

Data sharing statement All data relevant to the given manuscript have been stored in a separate file that can be made freely available to external investigators on request.

Open Access This is an Open Access article distributed in accordance with the Creative Commons Attribution Non Commercial (CC BY-NC 4.0) license, which permits others to distribute, remix, adapt, build upon this work non-commercially, and license their derivative works on different terms, provided the original work is properly cited and the use is non-commercial. See: http://creativecommons.org/ licenses/by-nc/4.0/

C) Article author(s) (or their employer(s) unless otherwise stated in the text of the article) 2018. All rights reserved. No commercial use is permitted unless otherwise expressly granted.

\section{REFERENCES}

1. GBD 2016 Causes of Death Collaborators. Global, regional, and national age-sex specific mortality for 264 causes of death, 19802016: a systematic analysis for the Global Burden of Disease Study 2016. Lancet 2017:390:1151-210.
2. Stroke Control Project Committee of National Health and Family Planning Commission of the People's Republic of China. Report on the Chinese stroke prevention (2015). 1st edn. Beijing: China Pecking Union Medical College Press, 2015.

3. Yang G, Wang Y, Zeng Y, et al. Rapid health transition in China, 19902010: findings from the global burden of disease study 2010. Lancet 2013;381:1987-2015.

4. Hata J, Kiyohara Y. Epidemiology of stroke and coronary artery disease in Asia. Circ J 2013;77:1923-32.

5. Mi T, Sun S, Du Y, et al. Differences in the distribution of risk factors for stroke among the high-risk population in urban and rural areas of Eastern China. Brain Behav 2016;6:e00461.

6. O'Donnell MJ, Xavier D, Liu L, et al. Risk factors for ischaemic and intracerebral haemorrhagic stroke in 22 countries (the INTERSTROKE study): a case-control study. Lancet 2010;376:112-23.

7. Wang J, Wen X, Li W, et al. Risk factors for stroke in the Chinese population: a systematic review and meta-analysis. J Stroke Cerebrovasc Dis 2017;26:509-17.

8. Feigin VL, Roth GA, Naghavi M, et al. Global burden of stroke and risk factors in 188 countries, during 1990-2013: a systematic analysis for the global burden of disease Study 2013. Lancet Neurol 2016;15:913-24.

9. Zhang PH, Jiao SJ, Zhou Y, et al. [Studies on prevalence and control of several common chronic diseases among Beijing adults in 2005]. Zhonghua Liu Xing Bing Xue Za Zhi 2007;28:625-30.

10. Sacco RL, Kasner SE, Broderick JP, et al. An updated definition of stroke for the 21st century: a statement for healthcare professionals from the American Heart Association/American Stroke Association. Stroke 2013;44:2064-89.

11. Chinese Diabetes Society. 2014 Chinese guidelines for secondary prevention of ischemic stroke and transient ischemic attack. Chinese Journal of Diabetes 2012;20:1-36.

12. China hypertension prevention guidelines revision committee. Guidelines for prevention and treatment of hypertension in China[2010 Edition]. Chinese Journal of hypertension 1919;2011:701-43.

13. Zhou BF. Predictive values of body mass index and waist circumference for risk factors of certain related diseases in Chinese adults--study on optimal cut-off points of body mass index and waist circumference in Chinese adults. Biomed Environ Sci 2002;15:83-96.

14. Hosmer DW, Lemeshow S. Confidence interval estimation of interaction. Epidemiology 1992;3:452-6.

15. Knol MJ, van der Tweel I, Grobbee DE, et al. Estimating interaction on an additive scale between continuous determinants in a logistic regression model. Int J Epidemiol 2007;36:1111-8.

16. Knol MJ, VanderWeele TJ, Groenwold RH, et al. Estimating measures of interaction on an additive scale for preventive exposures. Eur $J$ Epidemiol 2011;26:433-8.

17. Pan $\mathrm{Y}$, Wang $\mathrm{Y}$, Li H, et al. Association of diabetes and prognosis of minor stroke and its subtypes: a prospective observational study. PLoS One 2016;11:e0153178.

18. Tsai CF, Anderson N, Thomas B, et al. Risk factors for ischemic stroke and its subtypes in Chinese vs. Caucasians: systematic review and meta-analysis. Int J Stroke 2015;10:485-93.

19. Guo Y, Wang H, Tao T, et al. Determinants and time trends for ischaemic and haemorrhagic stroke in a large Chinese population. PLoS One 2016;11:e0163171.

20. Shi FL, Hart RG, Sherman DG, et al. Stroke in the People's Republic of China. Stroke 1989;20:1581-5.

21. Chang T, Gajasinghe S, Arambepola C. Prevalence of stroke and its risk factors in Urban Sri Lanka: population-based study. Stroke 2015;46:2965-8.

22. Muñoz-Rivas N, Méndez-Bailón M, Hernández-Barrera $\mathrm{V}$, et al. Type 2 diabetes and hemorrhagic stroke: a population-based study in Spain from 2003 to 2012. J Stroke Cerebrovasc Dis 2016;25:1431-43.

23. Tsai CF, Thomas B, Sudlow CL. Epidemiology of stroke and its subtypes in Chinese vs white populations: a systematic review. Neurology 2013;81:264-72.

24. Folsom AR, Rasmussen ML, Chambless LE, et al. Prospective associations of fasting insulin, body fat distribution, and diabetes with risk of ischemic stroke. The Atherosclerosis Risk in Communities (ARIC) Study Investigators. Diabetes Care 1999;22:1077-83.

25. Iso $\mathrm{H}$, Imano $\mathrm{H}$, Kitamura $\mathrm{A}$, et al. Type 2 diabetes and risk of nonembolic ischaemic stroke in Japanese men and women. Diabetologia 2004;47:2137-44.

26. Peters SA, Huxley RR, Woodward M. Diabetes as a risk factor for stroke in women compared with men: a systematic review and metaanalysis of 64 cohorts, including 775,385 individuals and 12,539 strokes. Lancet 2014;383:1973-80. 
27. Liao CC, Shih CC, Yeh CC, et al. Impact of diabetes on stroke risk and outcomes: two nationwide retrospective cohort studies. Medicine 2015;94:e2282.

28. Robson R, Lacey AS, Luzio SD, et al. HbA1c measurement and relationship to incident stroke. Diabet Med 2016;33:459-62.

29. Bragg F, Li L, Bennett $D$, et al. Association of random plasma glucose levels with the risk for cardiovascular disease among Chinese adults without known diabetes. JAMA Cardiol 2016;1:813-23.

30. Wu L, Wang A, Wang X, et al. Factors for short-term outcomes in patients with a minor stroke: results from China national stroke registry. BMC Neurol 2015;15:253.

31. Wang W, Jiang B, Sun $\mathrm{H}$, et al. Prevalence, incidence, and mortality of stroke in China: results from a nationwide population-based survey of 480687 adults. Circulation 2017;135:759-71.

32. Wolf PA, D'Agostino RB, Kannel WB, et al. Cigarette smoking as a risk factor for stroke. The Framingham Study. JAMA 1988;259:1025-9.

33. Wannamethee SG, Shaper AG, Whincup PH, et al. Smoking cessation and the risk of stroke in middle-aged men. JAMA 1995;274:155-60.

34. Qureshi Al, Suri MF, Kirmani JF, et al. Cigarette smoking among spouses: another risk factor for stroke in women. Stroke 2005;36:e74-e76.

35. Peters SA, Huxley RR, Woodward M. Smoking as a risk factor for stroke in women compared with men: a systematic review and metaanalysis of 81 cohorts, including 3,980,359 individuals and 42,401 strokes. Stroke 2013;44:2821-8.

36. Shah RS, Cole JW. Smoking and stroke: the more you smoke the more you stroke. Expert Rev Cardiovasc Ther 2010;8:917-32.

37. Chang S, Kim H, Kim V, et al. Association between smoking and physician-diagnosed stroke and myocardial infarction in male adults in Korea. Int J Environ Res Public Health 2016;13:158.

38. Soulimane S, Simon D, Herman WH, et al. HbA1c, fasting and $2 \mathrm{~h}$ plasma glucose in current, ex- and never-smokers: a meta-analysis. Diabetologia 2014;57:30-9.

39. Papademetriou V, Narayan $\mathrm{P}$, Rubins $\mathrm{H}$, et al. Influence of risk factors on peripheral and cerebrovascular disease in men with coronary artery disease, low high-density lipoprotein cholesterol levels, and desirable low-density lipoprotein cholesterol levels. HIT Investigators. Department of Veterans Affairs HDL Intervention Trial. Am Heart J 1998;136(Pt 1):734-40.

40. Aronson D, Rayfield EJ. How hyperglycemia promotes atherosclerosis: molecular mechanisms. Cardiovasc Diabetol 2002;1:1:1.

41. Rehni AK, Nautiyal N, Perez-Pinzon MA, et al. Hyperglycemia / hypoglycemia-induced mitochondrial dysfunction and cerebral ischemic damage in diabetics. Metab Brain Dis 2015;30:437-47.
42. Shukla V, Shakya AK, Perez-Pinzon MA, et al. Cerebral ischemic damage in diabetes: an inflammatory perspective. $J$ Neuroinflammation 2017;14:21.

43. Zhang Z, Yan J, Shi H. Hyperglycemia as a risk factor of ischemic stroke. J Drug Metab Toxicol 2013;4.153.

44. Kitada M, Zhang Z, Mima A, et al. Molecular mechanisms of diabetic vascular complications. J Diabetes Investig 2010;1:77-89.

45. Kemeny SF, Figueroa DS, Clyne AM. Hypo- and hyperglycemia impair endothelial cell actin alignment and nitric oxide synthase activation in response to shear stress. PLoS One 2013;8:e66176.

46. Iida $\mathrm{M}$, lida $\mathrm{H}$, Dohi $\mathrm{S}$, et al. Mechanisms underlying cerebrovascular effects of cigarette smoking in rats in vivo. Stroke 1998;29:1656-65.

47. Barua RS, Ambrose JA, Srivastava S, et al. Reactive oxygen species are involved in smoking-induced dysfunction of nitric oxide biosynthesis and upregulation of endothelial nitric oxide synthase: an in vitro demonstration in human coronary artery endothelial cells. Circulation 2003;107:2342-7.

48. Benowitz NL. Cigarette smoking and cardiovascular disease: pathophysiology and implications for treatment. Prog Cardiovasc Dis 2003;46:91-111.

49. Chen S, Wu P, Zhou L, et al. Relationship between increase of serum homocysteine caused by smoking and oxidative damage in elderly patients with cardiovascular disease. Int J Clin Exp Med 2015;8:4446-54.

50. Prasad K, Dhar I, Caspar-Bell G. Role of advanced glycation end products and its receptors in the pathogenesis of cigarette smokeinduced cardiovascular disease. Int J Angiol 2015;24:75-80.

51. Ottum MS, Mistry AM. Advanced glycation end-products: modifiable environmental factors profoundly mediate insulin resistance. $J$ Clin Biochem Nutr 2015;57:1-12.

52. Biswas SK, Mudi SR, Mollah FH, et al. Serum soluble receptor for advanced glycation end products (SRAGE) is independently associated with cigarette smoking in non-diabetic healthy subjects. Diab Vasc Dis Res 2013;10:380-2.

53. Zhong C, Zhong X, Xu T, et al. Combined effects of hypertension and heart rate on the risk of stroke and coronary heart disease: a population-based prospective cohort study among Inner Mongolians in China. Hypertens Res 2015;38:883-8.

54. Du H, Li L, Bennett $\mathrm{D}$, et al. Fresh fruit consumption and major cardiovascular disease in China. N Engl J Med 2016;374:1332-43.

55. Ding L, Xu Y, Wang LM, et al. Smoking and its relation to metabolic status among Chinese adults: analysis of a nationwide survey. Biomed Environ Sci 2016;29:619-27.

56. Xu Y, Wang L, He J, et al. Prevalence and control of diabetes in Chinese adults. JAMA 2013;310:948-59. 